\title{
Engagement sensitive visual stimulation
}

\author{
Deepesh Kumar (1), Anirban Dutta (2), Abhijit Das (3), Uttama Lahiri (1)
}

(1) Electrical Engineering, Indian Institute of Technology Gandhinagar, India; (2) Leibniz Research Center for Working Environment and Human Factors, TU Dortmund, Germany; (3) Department of Neurorehabilitation, AMRI Institute of Neurosciences, Kolkata, India

This article is distributed under the terms of the Creative Commons Attribution Noncommercial License (CC BY-NC 4.0) which permits any noncommercial use, distribution, and reproduction in any medium, provided the original author(s) and source are credited.

\begin{abstract}
Stroke is one of leading cause of death and disability worldwide. Early detection during golden hour and treatment of individual neurological dysfunction in stroke using easy-to-access biomarkers based on a simple-to-use, cost-effective, clinically-valid screening tool can bring a paradigm shift in healthcare, both urban and rural. In our research we have designed a quantitative automatic home-based oculomotor assessment tool that can play an important complementary role in prognosis of neurological disorders like stroke for the neurologist. Once the patient has been screened for stroke, the next step is to design proper rehabilitation platform to alleviate the disability. In addition to the screening platform, in our research, we work in designing virtual reality based rehabilitation exercise platform that has the potential to deliver visual stimulation and in turn contribute to improving one's performance.
\end{abstract}

Key Words: stroke, virtual-reality, fixation, smooth-pursuit, balance rehabilitation

Eur J Transl Myol 2016; 26 (2): 145-149

Stroke is one of the leading causes of death and disability worldwide which makes stroke survivors heavily dependent on their caregiver for daily-living tasks. ${ }^{1}$ Early detection during golden hour and treatment of individual neurological dysfunction in stroke using easy-to-access biomarkers based on a simple-to-use, cost-effective, clinically-valid screening tool can bring a paradigm shift in healthcare, both urban and rural. Here, dysfunction of one's oculomotor system involved in goal-directed gaze data may present an easy-to-access biomarker where up to $86 \%$ of stroke patients have some form of oculomotor disturbance. ${ }^{2}$ In conventional practice, clinicians often use one's visual symptoms for prediction of stroke condition. The clinicians assess the health status of individuals with their naked eyes by detecting abnormality in eye movements, where the prediction is highly subjective in nature and dependent on the clinician's expertise. Additionally, restricted availability of trained clinicians especially in remotely located areas makes the situation worse. Hence, a quantitative automatic home-based oculomotor assessment tool may play an important adjunctive role even in presence of conventional neurological diagnosis. This can be achieved by presenting different types of visual stimulus in the form of virtual reality (VR) tasks to the participant which can trigger specific eye movements. The gaze-related metrices can be recorded by monitoring one's eye movement pattern in response to the visual task, thereby serving as biomarkers of one's oculomotor dysfunction.

Once the patient is screened and found to have neurological dysfunction with associated disability, the next step is to bring the patient back in community life through rehabilitation. Though in the first three months following stroke attack, a major part of neurological recovery is possible through user-specific rehabilitation services, yet, there is evidence from literature that the disability-related issues can also be addressed by using multidisciplinary rehabilitation approach even after three months of stroke onset. ${ }^{3}$ One of the important aspects of rehabilitation is repeated practice through exercise that can address one's disability problems considerably. Again expert physiotherapists tune their rehabilitation protocol based on the patient's capability to perform in an exercise task engagement in the task which can be subjective in nature. Thus it is critical to have quantitative estimate of one's task performance. Additionally the task need to be motivating and interesting to the patients thereby encouraging them to do repeated practice of exercise that is a limitation of conventional rehabilitation. ${ }^{4}$ Evidence from literature shows that exercise tasks which can provide a visual stimulation that can be representative of bio-feedback can be effective platform for rehabilitation. ${ }^{5}$ Among the different possibilities, we choose computer-based virtual reality platform as a cheap and user friendly 

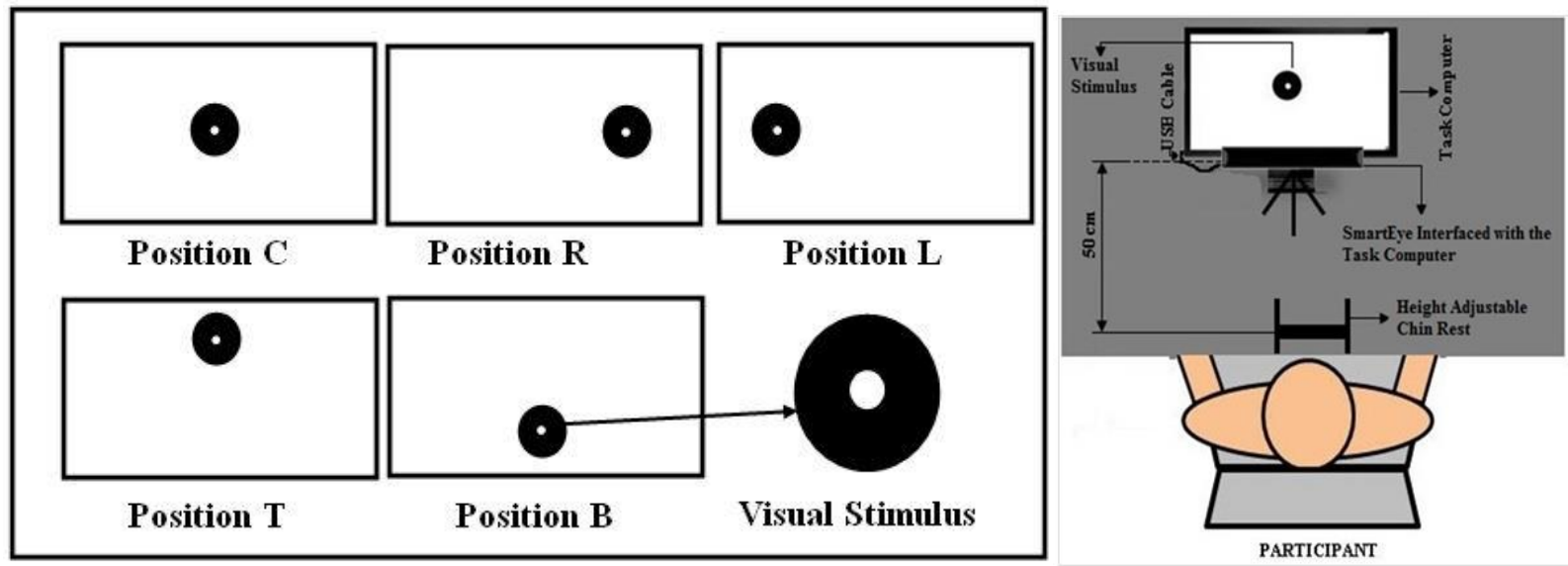

Fig 1. Left panel, Visual stimulation at various position; Right panel, Experimental Setup

medium of interaction by individuals with balance disorders. Here we choose balance as a target skill since balance is a critical requirement of one's daily living activities and is often adversely affected in patients with stroke. $^{6}$

Thus once the patients with neurological disorders have been screened by using oculometric parameters (as discussed above) we plan to expose the patients to VRbased balance skill learning platform and understand the implication of visual stimulation on balance rehabilitation. The rest of the paper is organized as follows: In the section Materials and Methods we present our system design and methodology. Results, discussion and future work follow.

\section{Materials and Methods}

\section{A. Post Stroke Eye Movement Evaluation}

Here we present a low-cost eye tracking system (SmartEye, henceforth) for quantitative assessment of oculomotor abnormalities when one is asked to participate in a visual task. As shown in Fig 1(left panel) we have presented a black circular visual stimulus appearing dynamically on a white background of $2 \mathrm{D}$ computer monitor to the participant. Figure 1 (right panel) shows the setup where a participant is asked to follow a dynamic visual stimulus presented on a computer screen. Simultaneously, SmartEye looks at the participant's eyes to detect gaze-related indices in a time synchronized manner. These indices related to fixation, smooth pursuit, etc. are used as biomarkers thereby detecting oculomotor abnormalities. Note that here the eye tracking calibration was performed by the experimenter himself. The experimenter's role in eye tracking calibration process was to offer 1) identical calibrated environment to all the participant and 2) a visual task environment in which the calibration was not adversely affected by gaze related abnormalities of the stroke survivor participants.

In our prior work we conducted a preliminary usability study involving 8 pairs of stroke and age-matched
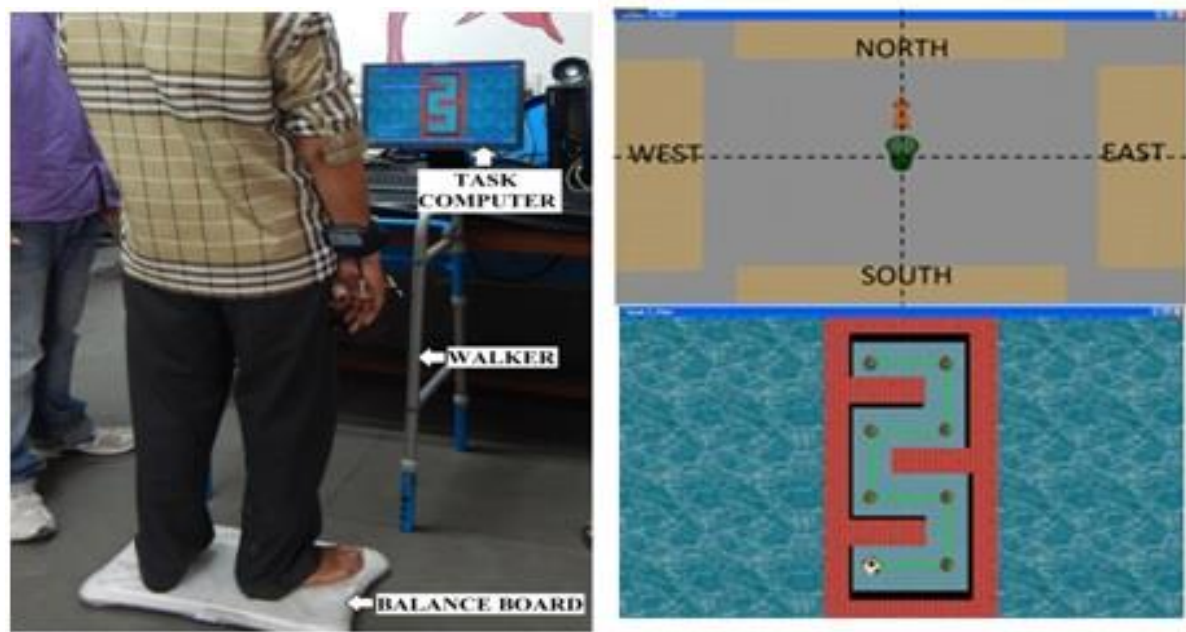

Fig 2. Left panel, Experimental setup; Right panel, GUIs of VR-based tasks. 

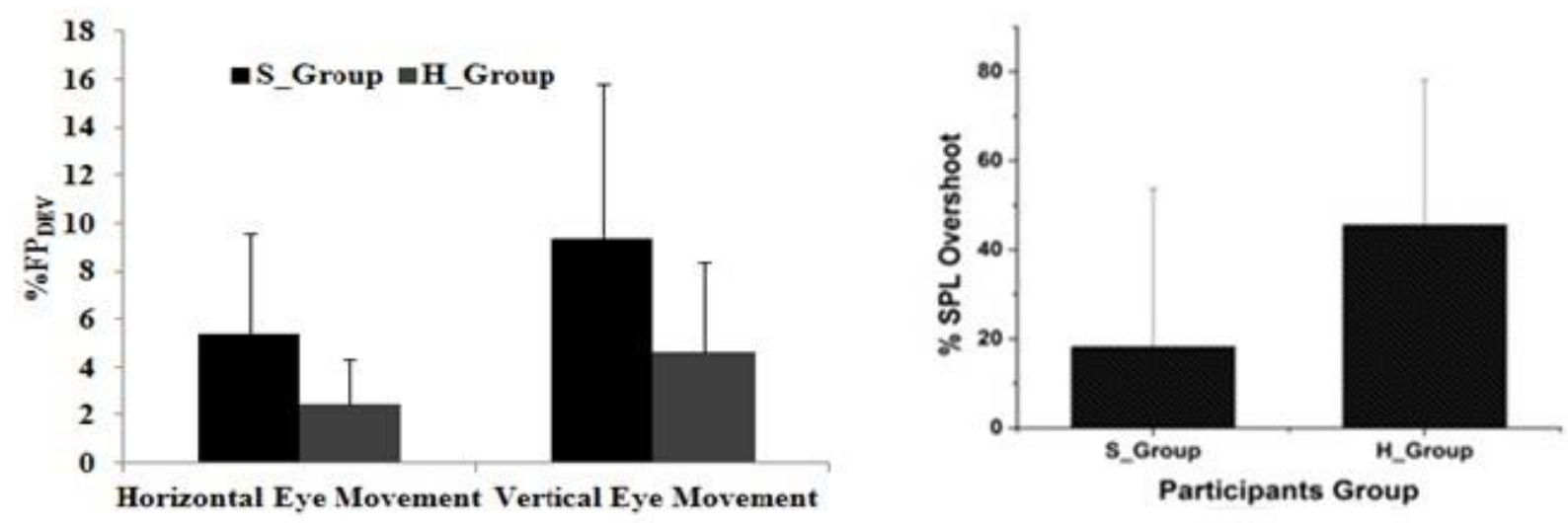

Fig 3. Left panel, Comparative group analysis of FPDEV for Healthy and Stroke participants; Right panel, Group average of Percentage Overshoot in SPL for Stroke and Healthy Groups.

healthy participants. ${ }^{7}$ Our aim was to understand whether it is possible to detect gaze-related indices that can be used as characteristic biomarkers of stroke condition while differentiating from the healthy individuals.

\section{B.VR-based Balance Rehabilitation Platform}

After the participant has been screened, we exposed the participant to a VR-based balance rehabilitation task. Here we detected the participant's center of pressure (CoP) which was integrated with a virtual object moving in a VR environment while the participant performed balance-related task. During the VR-based balance task, our system measured the participant's performance in a time-synched manner. Additionally, our system has the potential to pick up the participant's gaze-related indices in a time-synched manner that can be used to predict one's engagement during the task. We chose the balance task, since, one of the most common medical complications after stroke are falls with a reported incidence of up to $73 \%$ in the first year post-stroke. 8 Stroke-related ankle impairments including weakness of the ankle dorsiflexor muscles and increased spasticity of the ankle plantarflexor muscles lead to impaired postural control. Here, agility training programs can be leveraged that challenge dynamic balance where tasks are progressively increased in difficulty which may be more effective than static stretching/weight-shifting exercise program in preventing falls. Left panel of Fig 2 shows the experimental setup for lower limb rehabilitation exercise platform. The participant was asked to stand on a Balance Board (BB) kept in front of the Task Computer (PC). The BB was connected to task computer and measured the participant's CoP data while he performed the tasks shown on the computer monitor. Right panel of Fig. 2 shows an example of the VR-based balance rehabilitation task. The tasks in each difficulty level had different maximum possible scores allocated, namely, 900 points each for DL1 and DL2 and 700 points for DL3. We computed percentage performance score of each participant to take care of the different maximum possible scores of VR-based tasks belonging to DL1-DL3. In order to carry out a comparative analysis between the performance scores of the participants between Day $_{F}$ and Day $_{L}$, we needed to normalize their performance scores across sessions. The normalized weighted performance score was calculated as follows:

N_s $=(1 / 6 \mathrm{x}+2 / 6 \mathrm{y}+3 / 6 \mathrm{z}) /\left(1 / 6 \mathrm{x} \_\max +2 / 6 \mathrm{y} \_\max +3 / 6\right.$ z_max )

where Ns= Normalized performance score; $\mathrm{x}, \mathrm{y}, \mathrm{z}=$ average \% performance score in DL1, DL2 and DL3 respectively; $x \max , y \max , \mathrm{zmax}=100 \%$. As an initial approximation, we considered weights for tasks belonging to DL1, DL2 and DL3 as 1, 2 and 3 respectively.

In our previous work we presented a low-cost VRbased rehabilitation exercise platform developed as a proof-of-concept application. ${ }^{9}$ The difficulty level of the presented task can be decided based on the task conditions of the VR-based tasks. As shown in right panel of fig 2, in DL1, the task required the participant to shift weight in the instructed direction (namely North, East ,South and West), while moving a virtual object in the VR environment to a predefined position and then hold the object in that position for one second. In DL2, the task was similar to DL1 but here the thresold angle of weight shifting was increased to make the task more difficult. Finally, DL3 task was a maze like path along which the participants had to manuover the VR object from start point to end point. To make it difficult, no instruction was provided to participant regarding the direction of weight shifting and also the participant was expected to complete the task as fast as possible in order to achieve adequete performance score. We designed 30 different templates in each difficulty level so that our system offered 


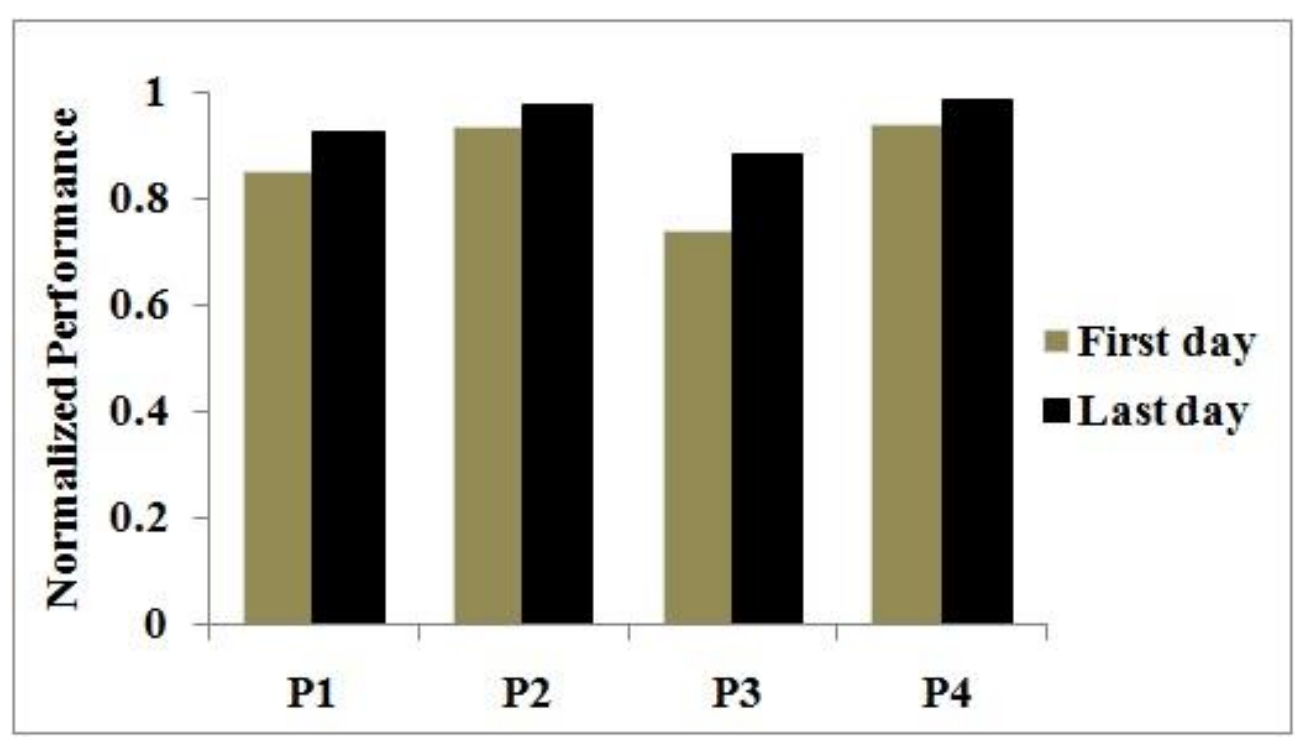

Fig. 4. Comparative Analysis of Normalized Performance Score on DayF and DayL

variations in task environment while the participants repeated task belonging to the same difficulty level untill his performance was adequate (except DL3 i.e. highest level). This VR-based excersise continued for 30 minutes.

\section{Results}

We designed usability studies as proof-of-concept applications using VR-based platform.

\section{A. Eye gaze based screening metric differentiating stroke} group from healthy group

To validate SmartEye as a user-friendly and easily accessible oculomotor monitoring tool that can be used to screen stroke from healthy group, we conducted a usability study with 8 stroke (S) and 8 age-matched healthy $(\mathrm{H})$ participants $[\mathrm{S}$ : mean $(\mathrm{SD})=55.87$ years (8.09 years); H: [mean $(\mathrm{SD})=59.87 \mathrm{y}(9.07)]$ and poststroke period $>4$ months. We found that the stroke group had shown higher deviation in their fixation capabilit (\% $\mathrm{FP}_{\mathrm{DEV}}$; implying incapability of fixating to a specific visual target appearing on the stimulus screen at discrete locations along the horizontal and vertical axes on the stimulus screen) than healthy group [Fig 3. (Left panel)]. Additionally, we also found that in the visual smooth pursuit task, mostly the stroke group could not follow the slow-moving visual target till the end of the trajectory on the computer monitor with some exceptions causing the average \% overshoot in the smooth pursuit length (SPL) being of the order of $20 \%$ (Fig. 3(Right panel)). In contrast, the healthy group often considerably overshooted the end point of the trajectory of the visual target possibly due to fast micro-saccades while tracking the slow-moving visual stimulus (Fig. 3, right panel).

\section{B. Implication of our VR-based system providing visual-stimulation on balance performance}

Here we present our findings of a preliminary usability study using VR-based platform providing visual stimulation for patients with balance disorders. As mentioned in our prior work on lower limb rehabilitation, designed as a proof-of-concept application, we conducted a usability study on 4 hemiparetic volunteers (P1-P4) [mean (SD) $=55.25$ years (4.78 years)], who were attending a rehabilitation program at a local civil hospital (Verma $\mathrm{S}$ et al, submittedpaper). Different participants interacted with different combinations of tasks in varying difficulty levels. We computed the normalized weighted performance scores of the participants. Fig. 4 shows the normalized performance scores for P1-P4 on the first day of rehabilitation $\left(\mathrm{Day}_{\mathrm{F}}\right)$ and the last day of rehabilitation $\left(\mathrm{Day}_{\mathrm{L}}\right)$. We find that for all the participants, there was an improvement in their normalized performance score from $\operatorname{Day}_{F}$ to Day . $_{\text {. }}$

\section{Implication of visual stimulation as evident from participats' feedback}

Our participants could visualize their weight shifting through displacement of the VR object that was mapped to their $\mathrm{CoP}$ measure. At the end of the excersise task on each day, the experimenter conducted an exit survey. The participant were asked i) whether they liked interacting with our system and (ii) if so, the reason behind their likes/dislikes. All the participant responded to the survey question by saying that they liked interacting with our system and they found it motivating due to the variations in the task environment that was presented to them as visual stimulus on the computer monitor. Additionally they 


\section{Engagement sensitive visual stimulation}

Eur J Transl Myol 26 (2): 145-149

said that they found the tasks interesting since they cn visualize their balance through the dynamic VR object displayed on the computer screen.

\section{Discussion}

SmartEye system was designed as an easily-accessible and cost-effective system to provide quantitative measures of gaze-related indices as biomarkers of neurological dysfunction such as stroke. Here we have first presented the use of SmartEye as a user-friendly and easily accessible oculomotor monitoring tool that can be employed for screening of oculomotor dysfunction, such as, in chronic stroke patients. Additionally, our previous studies have shown the efficacy of using gaze-related indices as predictors of one's engagement during a task. Subsequently, we presented our preliminary findings of a usability study that we carried out to understand the potential of visual stimulation delivered through VRbased balance tasks on improving participants' performance. However, here our VR-based balance exercise platform was sensitive to only one's performance during the task. With the possibility of mapping one's gaze indices to one's engagement level, in future, we plan to make our VR-based platform to provide not only visual stimulation to the patients, but also, make the platform sensitive to both the participant's individualized performance and predicted engagement level

generate safe and effective stimulations.

\section{Contributions}

Deepesh Kumar: Experiment design, data collection and analysis, publication writing; Anirban Dutta: Experiment design, Publication review; Abhijit Das: Experiment design, Publication review; Uttama Lahiri: Experiment design, supervision of data collection and analysis, publication drafting, publication review.

\section{Acknowledgements}

The authors would like to thank DST, India and INRIA, France for funding this research and also to the participants for making this study possible. We gratefully acknowledge the support of Spine Institute, Civil Medical Hospital, Ahmedabad, Gujarat, India.

\section{Conflict of Interest}

The author declare no potential conflict of interests.

\section{Corresponding Author}

Deepesh Kumar, Electrical Engineering, IIT Gandhinagar, Palaj, Simkheda, Gandhinagar-382355, Gujarat, India. E-mail: deepesh.kumar@iitgn.ac.in
E-mails of coAuthors

Anirban Dutta: nir.dutta@gmail.com

Abhijit Das: abhijit.neuro@gmail.com

Uttama Lahiri: uttamalahiri@iitgn.ac.in

\section{References}

1. Pandian JD, Sudhan P. Stroke Epidemiology and Stroke Care Services in India. J Stroke 2013;15:128-34. doi: 10.5853/jos.2013.15.3.128. Epub 2013 Sep 27

2. A. Pollock, C. Hazelton, C. A. Henderson et al., Interventions for disorders of eye movement in patients with stroke. Cochrane Database Syst Rev. 2012;3:CD008390. doi: 10.1002/14651858.CD 008390.pub2. Review.

3. MacDonald GA, Kayes NM, Bright F. Barriers and facilitators to engagement in rehabilitation for people with stroke: a review of the literature. New Zealand Journal of Physiotherapy 2013, 41: 112-121.

4. Saposnik G, Mindy L, Outcome Research Canada (SORCan) Working Group. Stroke Outcome Research Canada (SORCan) Working Group. Stroke 2011;42:1380-6. doi: 10.1161/STROKE AHA.110.605451. Epub 2011 Apr 7.

5. $\mathrm{Wu}, \mathrm{H}, \mathrm{Liu}, \mathrm{J}$, Handroos H, et al., Virtual reality based robotic therapy for stroke rehabilitation: An initial study. in Mechatronics and Automation (ICMA), International Conference on. IEEE 2011:1196-1200.

6. Tyson SF, Hanley M, Chillala J, Selley A, Tallis R.C. Balance disability after stroke. Physical therapy 2006; 86: 30-8.

7. Kumar D, Dutta A, Das A, Lahiri U. SmartEye: Developing a Novel Eye Tracking System for Quantitative Assessment of Oculomotor Abnormalities. Neural Systems and Rehabilitation Engineering, IEEE Transactions on 2016.

8. Verheyden, GS, Weerdesteyn V, Pickering RM, et al. Interventions for preventing falls in people after stroke. Cochrane Database Syst Rev 5.

9. D. Kumar, G., Aggarwal, R. Sehgal et al., Engagement-sensitive interactive neuromuscular electrical therapy system for post-stroke balance rehabilitation-a concept study. In Neural Engineering (NER), 2015 7th International IEEE/EMBS Conference on IEEE;190-3. 